\title{
Compatibility between pricing rules and risk measures: The CCVaR
}

\author{
Alejandro Balbás and Raquel Balbás
}

\begin{abstract}
This paper has considered a risk measure $\rho$ and a (maybe incomplete and/or imperfect) arbitrage-free market with pricing rule $\Pi$. They are said to be compatible if there are no reachable strategies $y$ such that $\Pi(y)$ is bounded and $\rho(y)$ is close to $-\infty$. We show that the lack of compatibility leads to meaningless situations in financial or actuarial applications.

The presence of compatibility is characterized by properties connecting the Stochastic Discount Factor of $\Pi$ and the sub-gradient of $\rho$. Consequently, several examples pointing out that the lack of compatibility may occur in very important pricing models are yielded. For instance the CVaR is not compatible with the Black and Scholes model or the CAPM.

We prove that for a given incompatible couple $(\Pi, \rho)$ we can construct a minimal risk measure $M_{(\Pi, \rho)}$ compatible with $\rho$ and such that $\rho \leq M_{(\Pi, \rho)}$. This result is particularized for the CVaR and the CAPM and the Black and Scholes model. Therefore we construct the Compatible Conditional Value at Risk (CCVaR). It seems that the CCVaR preserves the good properties of the CVaR and overcomes its shortcomings.
\end{abstract}

\section{Compatibilidad entre reglas de valoración y medidas de riesgo: el CCVaR}

Resumen. Consideraremos una medida de riesgo $\rho$ y un mercado libre de arbitraje (puede ser que incompleto o imperfecto) con regla de valoración П. Éstos serán compatibles si no hay estrategias alcanzables $y$ tales que $\Pi(y)$ permanece acotado y $\rho(y)$ se acerca a $-\infty$. Veremos que la falta de compatibilidad conduce a situaciones sin sentido económico en las aplicaciones actuariales o financieras.

La compatibilidad será caracterizada mediante propiedades que ligan al Factor de Descuento Estocástico de $\Pi$ y al sub-gradiente de $\rho$. Consecuentemente, se podrán dar importantes ejemplos en los que hay falta de compatibilidad. Por ejemplo, el CVaR no es compatible con el modelo de Black-Scholes o el CAPM.

Probaremos que para cualquier par incompatible $(\Pi, \rho)$ se puede construir una medida de riesgo minimal $M_{(\Pi, \rho)}$ compatible con $\rho$, y tal que $\rho \leq M_{(\Pi, \rho)}$. Este resultado se particularizará para el CVaR y el CAPM y el modelo de Black-Scholes. Por tanto, construiremos el CVaR Compatible (CCVaR). El CCVaR parece preservar las buenas propiedades del CVaR y superar sus deficiencias.

\section{Introduction}

General risk functions are becoming very important in finance and insurance. Since Artzner et al. [2, (1999)] introduced the axioms and properties of the "Coherent Measures of Risk" many authors have extended the

Palabras clave / Keywords: Risk measure, pricing rule, unbounded optimization problem, compatibility, compatible conditional value at risk.

Mathematics Subject Classifications: 91B28, 91B30. 
discussion. The recent development of new markets and products, the necessity of managing new types of risk, and the obligation of providing initial capital requirements have made it necessary to overcome the variance as the most used risk measure and to introduce more general risk functions. ${ }^{1}$ Hence, it is not surprising that the recent literature presents many interesting contributions focusing on new methods for measuring risk levels. Among others, Föllmer and Schied [10, (2002)] have defined the Convex Risk Measures, Goovaerts et al. [11, (2004)] have introduced the Consistent Risk Measures, Rockafellar et al. [16, (2006)] have defined the General Deviations and the Expectation Bounded Risk Measures, and Brown and Sim [7, (2009)] have introduced the Satisfying Measures. Further information about modern risk functions may be found in Balbás [3, (2007)].

Many classical actuarial and financial problems have been revisited by using new risk functions. So, with regard to portfolio choice and asset allocation problems, amongst many others authors, Alexander et al. [2, (2006)] compare the minimization of the Value at Risk (VaR) and the Conditional Value at Risk (CVaR) for a portfolio of derivatives, Calafiore [8, (2007)] studies "robust" efficient portfolios in discrete probability spaces, Mansini et al. [13, (2007)] use general risk measures in portfolio theory and Schied [19, (2007)] deals with optimal investment with convex risk measures.

Pricing and hedging issues in incomplete markets have also been studied (Föllmer and Schied [10, (2002)], Nakano, [14, (2004)], Staum, [20, (2004)], etc.), as well as Equity Linked Annuities hedging issues (Barbarin and Devolder [6, 2005]) and Optimal Reinsurance Problems (Balbás et al. [4], 2009). However, several optimization problems involving risk functions become unbounded, which does not make any sense in practical applications. It seems that this fact has not been deeply analyzed in the literature until now.

The present paper simultaneously considers the pricing rule of the market and the risk measurement procedure, and it points out that the "pathological" unbounded optimization problems may arise due to some lack of compatibility between the pricing rule and the risk function. In some sense, our major objective is to introduce and characterize the notion of compatibility between prices and risks, as well as to recover it when it does not hold.

The article's outline is as follows. Section 2 will present the notations and the general framework we are going to deal with. The concept of compatibility will be introduced in Section 3. We will consider a (maybe incomplete and/or imperfect) arbitrage-free market with pricing rule $\Pi$ and an expectation bounded risk measure $\rho$. They are compatible if there are no reachable strategies $y$ such that $\Pi(y)$ is bounded and $\rho(y)$ is close to $-\infty$ or, equivalently, there are no reachable strategies $y^{\prime}$ such that $\rho\left(y^{\prime}\right)$ is bounded and $\Pi\left(y^{\prime}\right)$ is close to $-\infty$. We will show that he lack of compatibility leads to meaningless situations in financial of actuarial applications. For instance, a manager could make the capital requirements disappear, borrow as much money as desired, and simultaneously face a riskless position, in the sense that the global risk of the strategy vanishes.

The most important result of this section is Theorem 1, which establishes that the necessary and sufficient condition to ensure compatibility is the existence of Stochastic Discount Factors of $\Pi$ in the subgradient of $\rho$. Accordingly, we will present several examples pointing out that the lack of compatibility may occur in very important pricing models. For instance, it happens if the sub-gradient of $\rho$ is composed of essentially bounded random variables and the Stochastic Discount Factor (SDF) is unbounded. Examples of risk measures satisfying the condition above are, among others, the CVaR and the Dual Power Transform (DPT) of Wang [21, (2000)]. Examples of pricing models are, amongst others, the Black and Scholes model and the Capital Asset Pricing Model (CAPM).

Section 4 is devoted to show that for a given incompatible couple $(\Pi, \rho)$ we can construct a minimal expectation bounded risk measure $M_{(\Pi, \rho)}$ compatible with $\rho$ and such that $\rho \leq M_{(\Pi, \rho)}$. The most important result of this section is Theorem 2, where $M_{(\Pi, \rho)}$ is constructed and profoundly analyzed. The possible coherence of $M_{(\Pi, \rho)}$ for a coherent $\rho$ is also studied.

We focus on concrete risk functions and pricing models on Section 5. In particular, we deal with the CVaR, the DPT, and the Absolute Deviation, as well as with the CAPM and the Black and Scholes

\footnotetext{
${ }^{1}$ It has been proved that the variance is not compatible with the Second Order Stochastic Dominance if asymmetries and/or heavy tails are involved (Ogryczak and. Ruszczynski, [15, 1999]).
} 
model. For them all we analyze the extension $M_{(\Pi, \rho)}$, and point out its major properties. Special attention is devoted to the $\mathrm{CVaR}$ because this expectation bounded and coherent risk measure is becoming very popular among researchers, managers and practitioners, due to its favorable properties. From the CVaR we apply the findings of Section 4 so as to build the Compatible Conditional Value at Risk (CCVaR), a new coherent and expectation bounded measure of risk compatible with the CAPM and the Black and Scholes model. Hence, it seems that the CCVaR preserves the good properties of the CVaR and overcomes its shortcomings.

Section 6 points out the most important conclusions of the paper.

\section{Preliminaries and notations}

Consider the probability space $(\Omega, \mathcal{F}, \mu)$ composed of the set of "states of the world" $\Omega$, the $\sigma$-algebra $\mathcal{F}$ and the probability measure $\mu$. Consider also a couple of conjugate numbers $p \in[1, \infty)$ and $q \in(1, \infty]$ (i.e., $1 / p+1 / q=1$ ). As usual $L^{p}\left(L^{q}\right)$ denotes the Banach space of $\mathbb{R}$-valued random variables $y$ on $\Omega$ such that $\mathbb{E}\left(|y|^{p}\right)<\infty, \mathbb{E}()$ representing the mathematical expectation $\left(\mathbb{E}\left(|y|^{q}\right)<\infty\right.$, or $y$ essentially bounded if $q=\infty)$. According to the Riesz Representation Theorem, we have that $L^{q}$ is the dual space of $L^{p}$.

Consider a time interval $[0, T]$, a subset $\mathcal{T} \subset[0, T]$ of trading dates containing 0 and $T$, and a filtration of $\sigma$-algebras $\left(\mathcal{F}_{t}\right)_{t \in \mathcal{T}}$ providing the arrival of information and such that $\mathcal{F}_{0}=\{\emptyset, \Omega\}$ and $\mathcal{F}_{T}=\mathcal{F}$.

Let us assume that $Y \subset L^{p}$ is a convex cone composed of super-replicable pay-offs, i.e., for every $y \in Y$ there exists at least one self-financing portfolio whose final pay-off is $S_{T} \geq y$. Denote by $\mathcal{S}(y)$ the family of such self-financing portfolios, and suppose that there exists

$$
\Pi(y)=\inf \left\{S_{0} ;\left(S_{t}\right)_{t \in \mathcal{T}} \in \mathcal{S}(y)\right\}
$$

for every $y \in Y$. We will say that $\Pi(y)$ is the price of $y$. The market will be said to be complete if for every $y \in L^{p}$ there exists $\left(S_{t}\right)_{t \in \mathcal{T}} \in \mathcal{S}(y)$ such that $S_{T}=y$, and incomplete otherwise. Besides, the market will be said to be perfect if $Y$ is a subspace of $L^{p}$ and $\Pi: Y \rightarrow \mathbb{R}$ is linear and continuous, and imperfect otherwise. In general, we will impose the natural conditions, sub-additivity

$$
\Pi\left(y_{1}+y_{2}\right) \leq \Pi\left(y_{1}\right)+\Pi\left(y_{2}\right)
$$

for every $y_{1}, y_{2} \in Y$, and positive homogeneity

$$
\Pi(\alpha y)=\alpha \Pi(y)
$$

for every $y \in Y$ and $\alpha \geq 0$. Consequently, $\Pi$ is a convex function. Finally, we will assume the existence of a riskless asset that does not generate any friction, i.e., almost surely constant random variables $y=k$ belong to $Y$ for every $k \in \mathbb{R}$, and there exists a risk-free rate $r_{f} \geq 0$ such that

$$
\Pi(k)=k \mathrm{e}^{-r_{f} T}
$$

holds. It is easy to see that (3) leads to

$$
\Pi(y+k)=\Pi(y)+k \mathrm{e}^{-r_{f} T}
$$

for every $y \in Y$ and $k \in \mathbb{R}$. Indeed $\Pi(y+k) \leq \Pi(y)+k \mathrm{e}^{-r_{f} T}$ is clear, and

$$
\Pi(y)=\Pi(y+k-k) \leq \Pi(y+k)+\Pi(-k)=\Pi(y+k)-k \mathrm{e}^{-r_{f} T}
$$

implies the opposite inequality.

Let

$$
\rho: L^{p} \longrightarrow \mathbb{R}
$$

be a general risk function that a trader uses in order to control the risk level of his final wealth at $T$. Assume that $\rho$ is continuous and satisfies: 
a)

$$
\rho(y+k)=\rho(y)-k
$$

for every $y \in L^{p}$ and $k \in \mathbb{R}$.

b)

$$
\rho(\alpha y)=\alpha \rho(y)
$$

for every $y \in L^{p}$ and $\alpha>0$.

c)

$$
\rho\left(y_{1}+y_{2}\right) \leq \rho\left(y_{1}\right)+\rho\left(y_{2}\right)
$$

for every $y_{1}, y_{2} \in L^{p}$.

d)

$$
\rho(y) \geq-\mathbb{E}(y)
$$

for every $y \in L^{p} .^{2}$

Particular interesting examples are the Conditional Value at Risk (CVaR) of Rockafellar et al. [16, (2006)], the Dual Power Transform (DPT) of Wang [21, (2000)] and the Wang Measure (Wang [21, (2000)]), among many others. Furthermore, following the original idea of Rockafellar et al. [16, (2006)] to identify their Expectation Bounded Risk Measures and their Deviation Measures, it is easy to see that

$$
\rho(y)=\sigma(y)-\mathbb{E}(y)
$$

is continuous and satisfies a), b), c) and d) if and only if $\sigma: L^{p} \rightarrow \mathbb{R}$ is a continuous deviation, that is, if $\sigma$ is continuous and satisfies $b$ ), c),

e)

$$
\sigma(y+k)=\sigma(y)
$$

for every $y \in L^{p}$ and $k \in \mathbb{R}$, and

f)

$$
\sigma(y) \geq 0
$$

for every $y \in L^{p}$.

Particular examples of deviation measures are the classical $p$-deviation given by

$$
\sigma(y)=\left[\mathbb{E}\left(|\mathbb{E}(y)-y|^{p}\right)\right]^{1 / p}
$$

or the downside $p$-semi-deviation given by

$$
\sigma(y)=\left[\mathbb{E}\left(|\max \{\mathbb{E}(y)-y, 0\}|^{p}\right)\right]^{1 / p},
$$

among many others.

Consider a continuous $\rho$ satisfying a), b), c) and d). Denote by

$$
\Delta_{\rho}=\left\{z \in L^{q} ;-\mathbb{E}(y z) \leq \rho(y), \forall y \in L^{p}\right\} .
$$

\footnotetext{
${ }^{2}$ Actually, the properties above are almost similar to those used by Rockafellar et al. [16, (2006)] in order to introduce their Expectation Bounded Risk Measures. These authors also impose a), b), c) and d), work with $p=2$, allow for $\rho(y)=\infty$, and impose $\rho(y)>-\mathbb{E}(y)$ if $y$ is not constant.
} 
The set $\Delta_{\rho}$ is obviously convex. Bearing in mind the Representation Theorem 2.4.9 in Zalinescu [22, (2002)] for convex functions, and using a proof similar to that of the Representation Theorem of risk measures stated in Rockafellar et al. [16, (2006)], it may be stated that $\Delta_{\rho}$ is also $\sigma\left(L^{q}, L^{p}\right)$-compact, the constant random variable $1 \in \Delta_{\rho}$, and

$$
\rho(y)=\max \left\{-\mathbb{E}(y z): z \in \Delta_{\rho}\right\}
$$

holds for every $y \in L^{p}$. Furthermore,

$$
\Delta_{\rho} \subset\left\{z \in L^{q} ; \mathbb{E}(z)=1\right\}
$$

Following Rockafellar et al. [16, (2006)], if $\rho$ is continuous and satisfies Properties a), b), c) and d) above then it is also coherent in the sense of Artzner et al. [2, (1999) $]^{3}$ if and only if

$$
\Delta_{\rho} \subset L_{+}^{q}=\left\{z \in L^{q} ; \mu(z \geq 0)=1\right\}
$$

Finally, by means of the Hahn Banach Separation Theorem, one may easily prove that if $\Delta \subset L^{q}$ is convex and $\sigma\left(L^{q}, L^{p}\right)$-compact, $1 \in \Delta$, and $\Delta$ satisfies (14), then there exists a unique continuous $\rho$ satisfying a), b), c) and d) such that (13) holds.

Summarizing, as indicated in the diagram below

$$
\begin{array}{ll}
\mathcal{D}_{\mu} & \leftrightharpoons \mathcal{M}_{\mu} \leftrightharpoons \mathcal{C}_{\mu} \\
\sigma=\rho+\mathbb{E} \leftrightharpoons \Delta_{\rho}
\end{array}
$$

Expression (9) establishes a one to one bijection between the set $\mathcal{M}_{\mu}$ of continuous functions satisfying a), b), c) and d) and the set $\mathcal{D}_{\mu}$ of continuous functions satisfying b), c), e) and f), whereas (13) (or (12)) establishes a one to one bijection between the set $\mathcal{M}_{\mu}$ and the set $\mathcal{C}_{\mu}$ of convex and $\sigma\left(L^{q}, L^{p}\right)$-compact subsets of $L^{q}$ fulfilling (14) and containing the constant random variable whose value is 1 . The coherence of the risk measure is characterized by the inclusion (15), and both identifications in (16) are increasing, i.e., higher deviations are associated with higher risk measures and higher sets of $\mathcal{C}_{\mu}$.

\section{Compatibility between pricing rules and risk measures}

This section will be devoted to introduce and characterize the notion of compatibility between risk measures and pricing rules.

Definition 1 The pricing rule $\Pi$ and the risk measure $\rho \in \mathcal{M}_{\mu}$ are said to be compatible if there are no sequences $\left(y_{n}\right)_{n=1}^{\infty} \subset Y$ such that $\Pi\left(y_{n}\right) \leq 0$ for every $n \in \mathbb{N}$ and

$$
\lim \rho\left(y_{n}\right)=-\infty
$$

simultaneously hold.

As some examples below will illustrate, the absence of compatibility may hold in practice.

Actually, if $\Pi$ and $\rho$ were not compatible, then every manager could make the capital requirements become $-\infty$, which does not make any sense in an economic framework. In fact, suppose that the random variable $y_{0} \in Y$ represents the value $T$ of the portfolio traded by the manager. Its final risk will be given by $\rho\left(y_{0}\right)$, which justifies that this quantity may be an adequate final value (at $T$ ) of the capital requirement. ${ }^{4}$ Indeed, (5) leads to

$$
\rho\left(y_{0}+\rho\left(y_{0}\right)\right)=0
$$

\footnotetext{
3i.e., $\rho\left(y_{1}\right) \geq \rho\left(y_{2}\right)$ whenever $y_{1}, y_{2} \in L^{p}$ and $y_{1} \leq y_{2}$.

${ }^{4}$ i.e., $\rho(y) \mathrm{e}^{-r} f_{f}^{T}$ should be the initial cash reserve (or capital requirement) invested in the risk-free asset.
} 
and the risk will vanish if the amount $\rho\left(y_{0}\right) \mathrm{e}^{-r_{f} T}$ is invested in the riskless security. But (7) and the existence of the sequence $\left(y_{n}\right)_{n=1}^{\infty} \subset Y$ above imply that

$$
\rho\left(y_{0}+y_{n}\right) \longrightarrow-\infty
$$

while

$$
\Pi\left(y_{0}+y_{n}\right) \leq \Pi\left(y_{0}\right),
$$

which means that no capital has to be added and the risk level may be reduced as desired if the manager buys $y_{n}$. Thus, the capital requirement $\rho\left(y_{0}\right)$ does not have to be added. On the contrary, by adding $y_{n}$ the trader may even borrow an arbitrary amount of money $-\rho\left(y_{0}+y_{n}\right) \rightarrow \infty$, since, according to (5),

$$
\rho\left(y_{0}+y_{n}+\rho\left(y_{0}+y_{n}\right)\right)=0 .
$$

Analogously, the lack of compatibility would allow an arbitrary trader to borrow an unbounded amount of money without facing any risky position. Indeed, borrowing $-\rho\left(y_{n}\right) \rightarrow \infty$ euros and buying $y_{n}$ for $\Pi\left(y_{n}\right) \leq 0$ euros would imply a global risk given by

$$
\rho\left(y_{n}+\rho\left(y_{n}\right)\right)=0,
$$

that must be interpreted as a null level of risk.

Next we will show that the inequality $\Pi\left(y_{n}\right) \leq 0$ may be substituted by a more general one.

Proposition 1 The pricing rule $\Pi$ and the risk measure $\rho \in \mathcal{M}_{\mu}$ are not compatible if and only if for every $a \in \mathbb{R}$ there exists a sequence $\left(y_{n}\right)_{n=1}^{\infty} \subset Y$ such that $\Pi\left(y_{n}\right) \leq$ a for every $n \in \mathbb{N}$ and (17) simultaneously hold.

Proof. Suppose that $\Pi$ and $\rho$ are not compatible and take the sequence $\left(y_{n}\right)_{n=1}^{\infty} \subset Y$ of Definition 1 . Then, (3) leads to

$$
\Pi\left(y_{n}+a \mathrm{e}^{r_{f} T}\right) \leq a,
$$

while (5) leads to

$$
\rho\left(y_{n}+a \mathrm{e}^{r_{f} T}\right)=\rho\left(y_{n}\right)-a \mathrm{e}^{r_{f} T} \longrightarrow-\infty,
$$

which completes the proof.

Next let us prove that the risk measure and the pricing rule may interchange their roles in Definition 1.

Proposition 2 The pricing rule $\Pi$ and the risk measure $\rho \in \mathcal{M}_{\mu}$ are not compatible if and only if for every $a \in \mathbb{R}$ there exists a sequence $\left(y_{n}\right)_{n=1}^{\infty} \subset Y$ such that $\rho\left(y_{n}\right) \leq$ a for every $n \in \mathbb{N}$ and

$$
\lim \Pi\left(y_{n}\right)=-\infty
$$

simultaneously hold.

PROOF. If $\left(y_{n}\right)_{n=1}^{\infty} \subset Y$ satisfies the conditions above then (4) and (5) easily show that

$$
\left(y_{n}-\Pi\left(y_{n}\right) \mathrm{e}^{r_{f} T}\right)_{n=1}^{\infty} \subset Y
$$

satisfies the conditions of Definition 1.

Conversely, if $\left(y_{n}\right)_{n=1}^{\infty} \subset Y$ satisfies the conditions of Definition 1 then (4) and (5) easily show that

$$
\left(y_{n}+\rho\left(y_{n}\right)-a\right)_{n=1}^{\infty} \subset Y
$$

satisfies the conditions above.

The interpretation of Propositions 1 and 2 seems to be clear. If $\Pi$ and $\rho$ are incompatible then there is a significant lack of balance between prices and risks. This lack may provoke pathological situations, as described above, that cannot be accepted in economic applications. 
Next we will attempt to characterize the notion of compatibility by means of practical criteria. To this purpose we will consider the optimization problem

$$
\left\{\begin{array}{l}
\min \rho(y) \\
\Pi(y) \leq 0 \\
y \in Y
\end{array}\right.
$$

Problem (18) minimizes the attainable risk level with non-positive prices. Obviously, (18) is bounded if and only if $\Pi$ and $\rho$ are compatible.

Problem (18) is not differentiable because $\rho$ is not differentiable either. Recent literature has developed several optimization methods that may solve this caveat (see, among others, Ruszczynski and Shapiro [18, (2006)]). In this paper we will follow a procedure quite parallel to that used in Balbás et al. [4, (2009)] and [5, (2009)], where the authors use risk measures and deal with a mathematical programming problems related to actuarial and financial classic topics. Some duality linked properties and Theorem 1 below will not be proved due to their analogy with similar results of the mentioned papers.

In particular, bearing in mind (13) and following Balbás et al. [5, (2009)], (18) is equivalent to the infinite-dimensional linear optimization problem

$$
\begin{cases}\min \theta & \\ \theta+\mathbb{E}(y z) \geq 0, & \forall z \in \Delta_{\rho} \\ \Pi(y) \leq 0 & y \in Y \\ \theta \in \mathbb{R}, & \end{cases}
$$

$\theta \in \mathbb{R}$ and $y \in L^{p}$ being the decision variables. Furthermore, $y \in Y$ solves (18) if and only if there exists $\theta \in \mathbb{R}$ such that $(\theta, y)$ solves (19), in which case $\theta=\rho(y)$ holds.

Besides, following parallel developments to that presented in Balbás et al. [4, (2009)], one can show that Problem

$$
\begin{cases}\max 0 & \\ \lambda \Pi(y)-\mathbb{E}(y z) \geq 0, & \forall y \in Y \\ \lambda \in \mathbb{R}, \quad \lambda \geq 0, & z \in \Delta_{\rho}\end{cases}
$$

is the dual of (19), $\lambda \in \mathbb{R}$ and $z \in \Delta_{\rho}$ being the decision variables.

Proposition 3 If $(\lambda, z)$ is (20)-feasible then $\lambda=\mathrm{e}^{r_{f} T}$.

Proof. Constraint

$$
\lambda \Pi(y)-\mathbb{E}(y z) \geq 0, \quad \forall y \in Y
$$

implies that $\mathbb{E}(z)=\lambda \mathrm{e}^{-r_{f} T}$, since we can take $y=1$ and $y=-1$ and apply (3). Then (14) leads to $\lambda=\mathrm{e}^{r_{f} T}$.

As a consequence, (20) may be simplified to

$$
\left\{\begin{array}{l}
\max 0 \\
\mathrm{e}^{r_{f} T} \Pi(y)-\mathbb{E}(y z) \geq 0, \quad \forall y \in Y \\
z \in \Delta_{\rho}
\end{array}\right.
$$

Finally, following Balbás et al. [4, (2009)] and [5, (2009)], there is no duality gap between (18) and (21), and the following primal-dual relationship holds

Theorem 1 The three following conditions are equivalent:

a) $\Pi$ and $\rho$ are not compatible. 
b) Problem (18) is unbounded.

c) Problem (21) has no feasible solutions.

Remark 1 Those elements satisfying the first constraint in (21) will be called Stochastic Discount Factors (SDF) of П. Actually, this notion of SDF is less restrictive than the usual one in Mathematical Finance (Duffie [9, (1988)]), since the classic framework takes $p=2$ and a perfect market. However, it is worthwhile to point out that the classical SDF would satisfy the first constraint in (21).

Notice that Theorem 2 indicates that $\Pi$ and $\rho$ are compatible if and only if there are SDF of $\Pi$ in the sub-gradient $\Delta_{\rho}$ of $\rho$.

Finally, let us remark that for a perfect market the first constraint in (21) must also apply if -y replaces $y$, which implies that the constraint may by given by

$$
\mathbb{E}(y z)=\mathrm{e}^{r_{f} T} \Pi(y)
$$

for every $y \in Y$.

Corollary $1 \Pi$ and $\rho$ are compatible if and only if

$$
\rho(y) \geq-\Pi(y) \mathrm{e}^{r_{f} T}
$$

for every $y \in Y$.

Proof. Suppose that $\Pi$ and $\rho$ are compatible and take a SDF $z \in \Delta_{\rho}$. Take $y \in Y$. Then (13) and (23) imply that

$$
\rho(y) \geq-\mathbb{E}(y z) \geq-\mathrm{e}^{r_{f} T} \Pi(y) .
$$

Conversely, suppose that (23) holds. Then $\Pi(y) \leq 0$ obviously implies that $\rho(y) \geq 0$. Thus (18) cannot be unbounded, and $\Pi$ and $\rho$ are compatible.

\section{Example 1 (Example illustrating that the compatibility between pricing rules and risk mea- sures is not guaranteed) Consider $\Omega=\left\{\omega_{1} \omega_{2}\right\}, \mu\left(\omega_{1}\right)=0.1, \mu\left(\omega_{2}\right)=0.9$, and}

$$
\Pi(\alpha(1,1)+\beta(1,0))=\alpha+0.5 \beta .
$$

The example indicates that the risk-free rate vanishes and the risky asset with pay-off $(1,0)$ has a price equal to 0.5. Suppose that

$$
\Delta_{\rho}=\left\{\left(z_{1}, z_{2}\right) ; 0.1 z_{1}+0.9 z_{2}=1 \text { and } 0 \leq z_{i} \leq 2.5, i=1,2\right\} .
$$

According to Rockafellar et al. [16, (2006)] $\Delta_{\rho}$ corresponds to the Conditional Value at Risk with $0.6=$ $60 \%$ as the level of confidence. Notice that this simple model satisfies many "good properties". For instance, it is perfect and complete, and it is also arbitrage-free because

$$
z_{1}=5, \quad z_{2}=\frac{5}{9}
$$

is a positive SDF (Duffie [9, (1988)]). However, the conditions defining the (21)-feasible set are

$$
\left\{\begin{array}{l}
0.1 z_{1}=0.5 \\
0.1 z_{1}+0.9 z_{2}=1 \\
0 \leq z_{i} \leq 2.5, \quad i=1,2
\end{array}\right.
$$

and this set is obviously void. Thus, according to Theorem 1, we are facing incompatibility. 
Proposition 4 Suppose that (18) (or (19)) is bounded. Then so is (21), every (21)-feasible solution solves (21) and $y^{*} \in Y$ solves (18) if and only if $\Pi\left(y^{*}\right)=0$ and $\rho\left(y^{*}\right)=0$. In particular, $y^{*}=0$ solves (18).

PROOF. (21) must be bounded due to the classical relationships between primal and dual problems (Luenberger [12, (1969)]). If $y^{*} \in Y$ solves (18) the absence of duality gap shows that $\rho\left(y^{*}\right)=0$. Let us prove $\Pi\left(y^{*}\right)=0$. If $\left.\Pi\left(y^{*}\right)\right)=-a<0$ then

$$
\Pi\left(y^{*}+a \mathrm{e}^{r_{f} T}\right)=0,
$$

whereas

$$
\rho\left(y^{*}+a \mathrm{e}^{r_{f} T}\right)=\rho\left(y^{*}\right)-a<\rho\left(y^{*}\right),
$$

which implies that $y^{*}$ is not optimal and we have a contradiction.

Conversely $y^{*} \in Y, \Pi\left(y^{*}\right)=0$ and $\rho\left(y^{*}\right)=0$ imply that $y^{*}$ is (18)-feasible and (18) achieves its optimal value at $y^{*}$, so $y^{*}$ is optimal.

Remark 2 Example 1 points out that $\rho$ and $\Pi$ may be incompatible, but we can provide more interesting examples. To this purpose, for $0<\mu_{0}<1$ define the $\mathrm{VaR}_{\mu_{0}}$ of every random variable y by (Balbás [3, (2007)])

$$
\operatorname{VaR}_{\mu_{0}}(y)=-\inf \left\{\alpha \in \mathbb{R} ; \mu(y \leq \alpha)>1-\mu_{0}\right\} .
$$

Since $L^{p} \subset L^{1}$, suppose that $\rho$ may be extended to the whole space $L^{1}$. Important expectation bounded risk measures satisfy this condition. Among others, the DPT of Wang [21, (2000)], given by

$$
\operatorname{DPT}_{a}(y)=\int_{0}^{1} \operatorname{VaR}_{1-t}(y) g_{a}^{\prime}(t) \mathrm{d} t
$$

for every $y \in L^{1}, a>1$ being an arbitrary constant and

$$
g_{a}:(0,1) \longrightarrow(0,1)
$$

given by

$$
g_{a}(t)=1-(1-t)^{a},
$$

the $\mathrm{CVaR}_{\mu_{0}}$ given by

$$
\operatorname{CVaR}_{\mu_{0}}(y)=\frac{1}{1-\mu_{0}} \int_{0}^{1-\mu_{0}} \operatorname{VaR}_{1-t}(y) \mathrm{d} t
$$

for $0<\mu_{0}<1$ and $y \in L^{1}$, the weighted CVaR (WCVaR) given by a convex combination of several $\mathrm{CVaRs}$ with different confidence level, and the measure (9) if $\sigma$ is the 1-deviation (or absolute deviation) or the 1-down-side semi-deviation (or down-side absolute semi-deviation) (see (10) and (11)). In such a case (12) points out that $\Delta_{\rho} \subset L^{\infty}$, and therefore the elements in $\Delta_{\rho}$ are essentially bounded. But there are many important pricing models in Financial Economics whose SDF are not essentially bounded. For instance, the Black and Scholes model, where the SDF is unique because the market is complete and it is unbounded too, as pointed out in Wang [21, (2000)]. Another important example is the Heston model, which allows us to price derivatives in an stochastic volatility framework. ${ }^{5}$

Remark 3 Expressions (24) and (26) point out that

$$
\mathrm{CVaR}_{\mu_{0}}(y) \geq \operatorname{VaR}_{\mu_{0}}(y)
$$

\footnotetext{
${ }^{5}$ Notice that the SDF of Example 1 is in $L^{\infty}$, so there are much more cases generating incompatibility, i.e., the given conditions are only sufficient.
} 
for every $y \in L^{1}$, which implies that

$$
\lim \operatorname{VaR}_{\mu_{0}}\left(y_{n}\right)=-\infty
$$

for every sequence $\left(y_{n}\right) \subset L^{1}$ such that

$$
\lim \operatorname{CVaR}_{\mu_{0}}\left(y_{n}\right)=-\infty .
$$

Thus, if the couple ( $\left.\Pi, \mathrm{CVaR}_{\mu_{0}}\right)$ is not compatible then nor is $\left(\Pi, \mathrm{VaR}_{\mu_{0}}\right)$, in the sense that $\left(\Pi, \operatorname{VaR}_{\mu_{0}}\right)$ does not satisfy Definition 1. Similarly, if the couple (П, WCVaR) is not compatible then the same occurs if $\mathrm{WCVaR}$ is replaced by $\mathrm{WVaR} .{ }^{6}$

\section{Recovering compatibility}

Since Example 1 and the previous remarks show that compatibility may fail in very important cases, it is natural to analyze whether modifications of the risk measure allow us to recover some kind of balance.

Theorem 2 Fix the pricing rule $\Pi$ and a risk measure $\rho \in \mathcal{M}_{\mu}$. Suppose that there exists a continuous $\tilde{\Pi}: L^{p} \rightarrow \mathbb{R}$ extending $\Pi$ and satisfying (1) and (2). Then there exists $M_{(\Pi, \rho)} \in \mathcal{M}_{\mu}$ such that:

a) $\Pi$ and $M_{(\Pi, \rho)}$ are compatible, and $\rho \leq M_{(\Pi, \rho)}$.

b) $M_{(\Pi, \rho)}$ is minimal, i.e., if $\Pi$ and $\tilde{\rho} \in \mathcal{M}_{\mu}$ are compatible, $\rho \leq \tilde{\rho}$ and $\tilde{\rho} \leq M_{(\Pi, \rho)}$ then $\tilde{\rho}=M_{(\Pi, \rho)}$.

c) $\Pi$ and $\rho$ are compatible if and only if $M_{(\Pi, \rho)}=\rho$.

d)

$$
M_{(\Pi, \rho)}(y) \geq \max \left\{-\Pi(y) \mathrm{e}^{r_{f} T}, \rho(y)\right\}
$$

holds for every $y \in Y$.

e) If the market is perfect (i.e., if $\Pi$ is linear and continuous) then

$$
M_{(\Pi, \rho)}(y)=\max \left\{-\Pi(y) \mathrm{e}^{r_{f} T}, \rho(y)\right\}
$$

holds for every $y \in Y$.

f) If the market is complete and perfect and the pricing rule $\Pi$ is increasing then $M_{(\Pi, \rho)}$ is coherent if and only if $\rho$ is coherent.

Proof. Consider the subspace $L \subset Y$ of $L^{p}$ composed of the constant functions and define $f(k)=$ $k \mathrm{e}^{-r_{f} T}$ for every $k \in L$. Bearing in mind (3), Theorem 3.2 in Rudin [17, (1972)] guarantees the existence of a linear function $\Lambda: L^{p} \rightarrow \mathbb{R}$ such that

$$
\Lambda(k)=k \mathrm{e}^{-r_{f} T}
$$

for every $k \in L$ and

$$
-\tilde{\Pi}(-y) \leq \Lambda(y) \leq \tilde{\Pi}(y)
$$

for every $y \in L^{p}$. The continuity of $\tilde{\Pi}$ and (29) obviously imply the continuity of $\Lambda$. Hence, the Riesz Theorem guarantees the existence of $z_{0} \in L^{q}$ such that $\Lambda(y) \mathrm{e}^{r_{f} T}=\mathbb{E}\left(y z_{0}\right)$ for every $y \in L^{p}$. Thus, (29) shows that

$$
\mathbb{E}\left(y z_{0}\right) \leq \tilde{\Pi}(y) \mathrm{e}^{r_{f} T}
$$

for every $y \in L^{p}$, and (28) shows that $\mathbb{E}\left(z_{0}\right)=1(k=1)$.

\footnotetext{
${ }^{6}$ It may be worth to recall now that VaR and WVaR are not expectation bounded because they are not sub-additive.
} 
It is easy to see that the set

$$
\tilde{\Delta}=\left\{t z+(1-t) z_{0} ; 0 \leq t \leq 1, z \in \Delta_{\rho}\right\}
$$

is convex, $\sigma\left(L^{q}, L^{p}\right)$-compact, and is composed of random variables whose expectation is one. Thus, $\tilde{\Delta} \in \mathcal{C}_{\mu}$.

Define

$$
\mathcal{R}=\left\{z \in L^{q} ; \mathbb{E}(z)=1 \text { and } \mathbb{E}(y z) \leq \tilde{\Pi}(y) \mathrm{e}^{r_{f} T} \quad \forall y \in L^{p}\right\}
$$

and let us prove that

$$
\mathcal{C}_{\mu}^{*}=\left\{\Delta \in \mathcal{C}_{\mu} ; \Delta \cap \mathcal{R} \neq \emptyset \text { and } \Delta_{\rho} \subset \Delta\right\}
$$

is inductive (with the opposite order, i.e., $\Delta_{1} \geq \Delta_{2}$ if $\Delta_{1} \subset \Delta_{2}$ ). Indeed, $\tilde{\Delta} \in \mathcal{C}_{\mu}^{*}$ implies that $\mathcal{C}_{\mu}^{*}$ is not empty, and the intersection of the elements in a chain of $\mathcal{C}_{\mu}^{*}$ is obviously convex, $\sigma\left(L^{q}, L^{p}\right)$-compact, composed of random variables whose expectation equals one, and contains $\Delta_{\rho}$. Moreover, this intersection has elements of $\mathcal{R}$ because $\mathcal{R}$ is (weakly*) closed, and a finite intersection in the chain obviously contains elements in $\mathcal{R}$.

The Zorn's Lemma implies the existence of a minimal element $\Delta \in \mathcal{C}_{\mu}^{*}$ such that $\Delta \subset \tilde{\Delta}$. According to (16) and (13) $\Delta$ defines a risk measure $M_{(\Pi, \rho)} \in \mathcal{M}_{\mu}$, and (13) and $\Delta_{\rho} \subset \Delta$ imply that $\rho \leq M_{(\Pi, \rho)}$. Moreover b) holds because $\Delta$ is minimal in $\mathcal{C}_{\mu}^{*}$, and the identification of (16) conserves the natural order.

Property c) trivially follows from a) and b) if one takes $\tilde{\rho}=\rho$.

Property d) trivially follows from a) and Corollary 1 . To see e), i.e., the opposite inequality in a perfect market, fix $y \in Y . \Delta \subset \tilde{\Delta}$ implies that

$$
M_{(\Pi, \rho)}(y) \leq \max \{-\mathbb{E}(y z): z \in \tilde{\Delta}\} .
$$

Take $z_{2} \in \tilde{\Delta}$ where the maximum above is reached and $z_{1} \in \Delta_{\rho}$ and $t \in[0,1]$ such that

$$
z_{2}=t z_{1}+(1-t) z_{0}
$$

Since the market is perfect, (30) and (22) lead to

$$
\begin{aligned}
M_{(\Pi, \rho)}(y) & \leq-t \mathbb{E}\left(y z_{1}\right)-(1-t) \mathbb{E}\left(y z_{0}\right) \\
& \leq t \rho(y)-(1-t) \Pi(y) \mathrm{e}^{r_{f} T} \\
& \leq \max \left\{-\Pi(y) \mathrm{e}^{r_{f} T}, \rho(y)\right\}
\end{aligned}
$$

Finally, to see f), from (27) it trivially follows that $M_{(\Pi, \rho)}$ is decreasing if so are $-\Pi$ and $\rho$. Conversely, if $M_{(\Pi, \rho)}$ is coherent then its associate set $\Delta \in \mathcal{C}_{\mu}$ is composed of non-negative random variables (see (15)), and therefore so is $\Delta_{\rho} \subset \Delta$.

Remark 4 Notice that the existence of the extension $\tilde{\Pi}$ above frequently holds. For instance, if the market is perfect, i.e., if $Y$ is a subspace and $\Pi$ is linear and continuous, then the existence of $\tilde{\Pi}$ follows from the Hahn Banach Theorem. On the other hand, if the market is complete and perfect then $\Pi$ will be increasing so as to prevent the existence of arbitrage (Duffie [9, (1988)]), i.e., Property e) applies.

\section{Modified risk measures: The CCVaR}

Now we are in a position to revisit Example 1 and those important cases of Remarks 2 and 3.

With respect to Example 1, we are dealing with a complete market, so we can take $\tilde{\Pi}=\Pi$ in the latter theorem. Thus we have a modified $\mathrm{CVaR}_{0.6}$ that we will denote by $\mathrm{CCVaR}_{(\Pi, 0.6)}$ and will call "Compatible $\mathrm{CVaR}_{0.6}$ ". It is a new risk measure that retrieves compatibility with the pricing rule. Therefore 
it prevents the unbalanced pathological situations of Propositions 1 and 2. In some sense, the new risk measure retrieves some kind of balance between the $\mathrm{CVaR}_{0.6}$ and the pricing rule.

Besides, according to (27)

$$
\operatorname{CCVaR}_{(\Pi, 0.6)}(\alpha+\beta, \alpha)=\max \left\{-\Pi(\alpha+\beta, \alpha), \operatorname{CVaR}_{0.6}(\alpha+\beta, \alpha)\right\}
$$

for every $\alpha, \beta \in \mathbb{R}, \mathrm{CCVaR}_{(\Pi, 0.6)} \geq \mathrm{CVaR}_{0.6}, \mathrm{CCVaR}_{(\Pi, 0.6)}$ is expectation bounded and coherent, and $\mathrm{CCVaR}_{(\Pi, 0.6)}$ and $\Pi$ are compatible.

More generally we can take the general probability space $(\Omega, \mathcal{F}, \mu)$ and the general CVaR measure $\rho=\mathrm{CVaR}_{\mu_{0}}, \mu_{0} \in(0,1)$ being the level of confidence. According to Rockafellar et al. [16, (2006)] we have that

$$
\Delta_{\mathrm{CVaR}_{\mu_{0}}}=\left\{z \in L^{\infty} ; \mathbb{E}(z)=1,0 \leq z \leq \frac{1}{1-\mu_{0}}\right\} .
$$

Hence, bearing in mind (15), $\mathrm{CVaR}_{\mu_{0}}$ is a coherent and expectation bounded measure of risk. This property has provoked that $\mathrm{CVaR}_{\mu_{0}}$ is becoming a very popular risk measure for both researchers and practitioners, and it has been used to revisit many classical financial and actuarial problems (Alexander et al. [1, (2006)], Mansini et al. [13, (2007)], Balbás et al. [4, (2009)], etc.). However, since $\Delta_{\mathrm{CVaR}_{\mu_{0}}} \subset L^{\infty}$ the caveat of Remark 2 applies, i.e., $\mathrm{CVaR}_{\mu_{0}}$ is not compatible with the pricing rule of complete and perfect market models whose SDF is unbounded (Black and Scholes model, Heston model etc.). Nevertheless, according to Theorem 2, in these kind of models there is a minimal expectation bounded risk measure $\mathrm{CCVaR}_{\left(\Pi, \mu_{0}\right)}$ that will be called "Compatible $\mathrm{CVaR}_{\mu_{0}}$ " and satisfies

$$
\operatorname{CVaR}_{\mu_{0}}(y) \leq \operatorname{CCVaR}_{\left(\Pi, \mu_{0}\right)}(y)
$$

for every $y \in L^{p}{ }^{7}$

$$
\operatorname{CCVaR}_{\left(\Pi, \mu_{0}\right)}(y)=\max \left\{-\Pi(y) \mathrm{e}^{r_{f} T}, \operatorname{CVaR}_{\mu_{0}}(y)\right\}
$$

for every $y \in L^{p}$ and $\operatorname{CCVaR}_{\left(\Pi, \mu_{0}\right)}$ is coherent and compatible with the pricing rule of the model.

There are perfect but incomplete arbitrage free pricing models such that the classical SDF is also unbounded. The most important one, but not the only one, is the CAPM, where the SDF is closely related to the Market Portfolio (Duffie [9, (1988)]). Since our concept of SDF is strictly weaker than the classical one then the absence of elements in (31) satisfying (22) is not guaranteed. However, it may hold, which makes the $\mathrm{CVaR}_{\mu_{0}}$ a measure reflecting a serious drawback. However, according to Theorem 2 and its remark, a Compatible CVaR may still be defined, and it is a minimal expectation bounded risk measure that also satisfies (32), and (33) holds for every reachable pay-off $y$. Moreover, $\operatorname{CCVaR}_{\left(\Pi, \mu_{0}\right)}$ is compatible with the pricing rule of the model, and, owing to Theorem $2 \mathrm{c}$ ), $\mathrm{CVaR}_{\mu_{0}}$ is compatible with the pricing rule if and only if $\mathrm{CVaR}_{\mu_{0}}=\mathrm{CCVaR}_{\left(\Pi, \mu_{0}\right)}$, which is consistent with (33) and Corollary 1. Finally, the element $z_{0}$ in the proof of Theorem 2 may be replaced by an alternative element of $L^{q}$ satisfying (22), that may be non-negative due to the absence of arbitrage. Then, bearing in mind (15) and following the same proof as in Theorem 2, but modifying the set $\mathcal{C}_{\mu}^{*}$ according to

$$
\mathcal{C}_{\mu}^{*}=\left\{\Delta \in \mathcal{C}_{\mu} ; \Delta \subset L_{+}^{q}, \Delta \cap \mathcal{R} \neq \emptyset \text { and } \Delta_{\rho} \subset \Delta\right\},
$$

$L_{+}^{q}$ denoting the usual non-negative cone of $L^{q}, \operatorname{CCVaR}_{\left(\Pi, \mu_{0}\right)}$ may be constructed in such a way that it is also coherent.

Let us remark that the role of the CVaR may be also played, amongst others, by the WCVaR, the DPT of (25) and the Absolute Deviation of (9) and (10) with $p=1$. Thus we can build the Compatible WCVaR, the Compatible DPT and the Compatible Absolute Deviation, denoted by CWCVaR, CDPT and CAD. Furthermore, as stated in Theorem 2, the construction above may also make sense for risk measures that cannot be extended to $L^{1}$.

\footnotetext{
${ }^{7}$ Actually, for the cited models $p=2$.
} 


\section{Conclusions}

This paper has considered an expectation bounded risk measure $\rho$ and an arbitrage-free market with pricing rule $\Pi$. They have been said to be compatible if there are no reachable strategies $y$ such that $\Pi(y)$ is bounded and $\rho(y)$ is close to $-\infty$ or, equivalently, there are no reachable strategies $y^{\prime}$ such that $\rho\left(y^{\prime}\right)$ is bounded and $\Pi\left(y^{\prime}\right)$ is close to $-\infty$. We have shown that the lack of compatibility leads to meaningless situations in financial or actuarial applications. For instance, a manager can borrow as much money as desired and simultaneously face a riskless position. Furthermore, incompatibility makes it unbounded several optimization problems with significant economic meaning.

Compatibility has been characterized by the existence of Stochastic Discount Factors (SDF) of $\Pi$ in the sub-gradient of $\rho$. Hence, several examples pointing out that the lack of compatibility may occur in very important pricing models have been yielded. For instance, it happens if the sub-gradient of $\rho$ is composed of essentially bounded random variables and the SDF is unbounded. Examples of risk measures are, among others, the CVaR and the DPT. Examples of pricing models are the Black and Scholes model, the Heston model and the CAPM.

We have proved that for a given incompatible couple $(\Pi, \rho)$ we can construct a minimal risk measure $M_{(\Pi, \rho)}$ compatible with $\Pi$ and such that $\rho \leq M_{(\Pi, \rho)}$. This result has been particularized for important risk functions and pricing models. In particular, we have dealt with the CVaR, the DPT, and the Absolute Deviation, as well as with the CAPM and the Black and Scholes model. For them all the extension $M_{(\Pi, \rho)}$ has been studied. Special attention was devoted to the CVaR because this expectation bounded and coherent risk measure is becoming very popular among researchers, managers and practitioners, due to its good properties. From the $\mathrm{CVaR}$ we have constructed the Compatible Conditional Value at Risk (CCVaR), a new coherent and expectation bounded measure of risk compatible with the CAPM and the Black and Scholes model. It seems that the CCVaR preserves the good properties of the CVaR and overcomes its shortcomings.

Acknowledgement. Research partially supported by "RD_Sistemas SA", "Comunidad Autónoma de Madrid" (Spain), Grant s-0505/tic/000230, and "MEyC” (Spain), Grant SEJ2006-15401-C04. The usual caveat applies.

\section{References}

[1] Alexander, S., Coleman, T. F. And Li, Y., (2006). Minimizing CVaR and VaR for a portfolio of derivatives, Journal of Banking \& Finance, 30, 538-605.

[2] Artzner, P., Delbaen, F., Eber, J. M. and Heath, D., (1999). Coherent measures of risk, Math. Finance, 9, 203-228.

[3] Balbás, A., (2007). Mathematical methods in modern risk measurement: A survey, RACSAM Rev. R. Acad. Cien. Serie A. Mat., 101(2), 205-219.

[4] Balbás, A., Balbás, B. And Heras, A., (2009). Optimal reinsurance with general risk measures, Insurance Math. Econom., 44, 374-384, doi: 10.1016/j.insmatheco.2008.11.008.

[5] Balbás, A., Balbás, R. And Mayoral, S., (2009). Portfolio choice problems and optimal hedging with general risk functions: A simplex-like algorithm, European J. Oper. Res., 192, 2, 603-620.

[6] Barbarin, J. And Devolder, P., (2005). Risk measure and fair valuation of an investment guarantee in life insurance, Insurance Math. Econom., 37, 2, 297-323.

[7] Brown, D. And Sim, M., (2009). Satisfying measures for analysis of risky positions, Management Science, 55, 71-84, doi: 10.1287mnsc.1080.0929.

[8] Calafiore, G. C., (2007). Ambiguous risk measures and optimal robust portfolios, SIAM J. Optim., 18, 3 , 853-877. 
[9] DufFIE, D., (1988). Security markets: Stochastic models, Academic Press.

[10] Föllmer, H. And Schied, A., (2002). Convex measures of risk and trading constraints, Finance Stoch., 6, 429-447.

[11] Goovaerts, M., KaAs, R., Dhaene, J. And TAng, Q., (2004). A new classes of consistent risk measures, Insurance Math. Econom., 34, 505-516.

[12] Luenberger, D. G., (1969). Optimization by vector spaces methods, John Wiley \& Sons, New York.

[13] Mansini, R., Ogryczak, W. and Speranza, M. G., (2007). Conditional value at risk and related linear programming models for portfolio optimization, Ann. Oper. Res., 152, 227-256.

[14] NaKano, Y., (2004). Efficient hedging with coherent risk measure, J. Math. Anal. Appl., 293, 345-354.

[15] Ogryczak, W. And Ruszczynski, A., (1999). From stochastic dominance to mean risk models: Semideviations and risk measures, European J. Oper. Res., 116, 33-50.

[16] Rockafellar, R. T., Uryasev, S. And Zabarankin, M., (2006). Generalized deviations in risk analysis, Finance Stoch., 10, 51-74.

[17] Rudin, W., (1972). Functional Analysis, McGraw-Hill Book Company, New York.

[18] Ruszczynski, A. And Shapiro, A., (2006). Optimization of convex risk functions, Math. Oper. Res., 31, 3, $433-452$.

[19] SchIED, A., (2007). Optimal investments for risk- and ambiguity-averse preferences: A duality approach, $\mathrm{Fi}$ nance Stoch., 11, 107-129.

[20] Staum, J., (2004). Fundamental theorems of asset pricing for good deal bounds, Math. Finance, 14, $141-161$.

[21] WANG, S. S., (2000). A class of distortion operators for pricing financial and insurance risks, Journal of Risk and Insurance, 67, 15-36.

[22] Zalinescu, C., (2002). Convex analysis in general vector spaces, World Scientific Publishing Co.

\section{Alejandro Balbás}

Académico Correspondiente de la

Real Academia de Ciencias.

University Carlos III of Madrid.

CL. Madrid, 126. 28903 Getafe,

Madrid, Spain.

alejandro.balbas@uc 3m.es

\section{Raquel Balbás}

Department of Actuarial and Financial Economics.

Somosaguas Campus.

University Complutense of Madrid

28223 Pozuelo de Alarcón,

Madrid, Spain.

raquel.balbas@ccee. ucm.es 